\title{
Erratum to: On additively regular seminearrings
}

\author{
Sujit Kumar Sardar ${ }^{1}$ • Rajlaxmi Mukherjee ${ }^{1}$
}

Received: 24 June 2015 / Accepted: 12 August 2015 / Published online: 2 September 2015

(C) Springer Science+Business Media New York 2015

\section{Erratum to: Semigroup Forum (2014) 88:541-554 DOI 10.1007/s00233-013-9538-z}

An error, in Section 3 of our paper [1], has come to our notice recently. This can be rectified firstly by replacing throughout the section 'full right $k$-ideal' and 'full $k$-ideal' by 'normal full right $k$-ideal' and 'normal full $k$-ideal', respectively. The definitions of normal full right $k$-ideal and normal full $k$-ideal can be given as follows and can be considered to be an extension of Definition 3.10 of [1].

Definition 3.10 (Extended [1]) In an additively inverse seminearring $(S,+$, ), a full right (left) $k$-ideal $H$ is said to be normal full right (left) $k$-ideal if $a+h+a^{*} \in H$ for all $h \in H$ and for all $a \in S$. The definition of normal full $k$-ideal is obvious.

The second step for the rectification of the error is accomplished by providing (see below) a minor correction in the proofs of Propositions 3.11 and 3.12. For other results such as Propositions 3.16 and 3.17, Theorems 3.19 and 3.20 the necessary corrections are obvious and so we omit them.

Communicated by Lev N. Shevrin.

The online version of the original article can be found under doi:10.1007/s00233-013-9538-z.

Sujit Kumar Sardar

sksardarjumath@gmail.com

Rajlaxmi Mukherjee

ju.rajlaxmi@gmail.com

1 Department of Mathematics, Jadavpur University, Kolkata 700032, India 
Proposition 3.11 ([1], corrected version) Let $S$ be an additively inverse seminearring and $H$ be a normal full right $k$-ideal of $S$. Then the relation $\rho$, defined on $S$, by

$a \rho b$ if and only if $a+b^{*} \in H$,

is a right normal congruence on $S$. Moreover $H=\{a \in S:$ ape for some e $\in$ $\left.E^{+}(S)\right\}$.

Proof Under the previous hypothesis $\rho$ was lacking the following property of being left compatible with respect to addition. Now we prove this as follows. Let $a \rho b$ and $c \in S$. Then $(c+a)+(c+b)^{*}=c+a+b^{*}+c^{*}$ where $a+b^{*} \in H$. Hence by the hypothesis $(c+a)+(c+b)^{*} \in H$ whence $(c+a) \rho(c+b)$.

Proposition 3.12 ([1], corrected version) Let $\rho$ be a right normal congruence on an additively inverse seminearring $S$. Then $H:=\left\{a \in S:\right.$ ape for some e $\left.\in E^{+}(S)\right\}$ is a normal full right $k$-ideal of $S$. Moreover, the relation $\sigma$, defined on $S$, by

$$
a \sigma b \text { if and only if } a+b^{*} \in H \text {, }
$$

coincides with $\rho$.

Proof It has already been proved in [1] that $H$ is a full right $k$-ideal of $S$. We now prove that $H$ is a normal full right $k$-ideal. Let $h \in H$. Then there exists $e \in E^{+}(S)$ such that $h \rho e$. Let $x \in S$. Then $x+e+x^{*} \in E^{+}(S)$. Now as $h \rho e,\left(x+h+x^{*}\right) \rho\left(x+e+x^{*}\right)$ using the compatibility of $\rho$ with respect to addition. Therefore $x+h+x^{*} \in H$.

Now for the other results of the said section [1] requiring correction we only provide below the corrected statements as the necessary corrections in the proof are easy to understand as they follow in a manner similar to that of either Proposition 3.11 or Proposition 3.12(corrected versions) given above.

Proposition 3.16 ([1], corrected version) Let $S$ be a distributively generated additively inverse seminearring with property $D$. Let $H$ be a normal full $k$-ideal of $S$. Then the relation $\sigma$, defined by

$a \sigma b$ if and only if $a+b^{*} \in H$ and $c a+(c b)^{*} \in H$ for all $c \in S$,

is a normal congruence on $S$. Moreover $H=\left\{a \in S:\right.$ a $\sigma$ e for some e $\left.\in E^{+}(S)\right\}$.

Proposition 3.17 ([1], corrected version) Let $S$ be a distributively generated additively inverse seminearring with property $D$ and let $\rho$ be a normal congruence on $S$. Then $H:=\left\{a \in S:\right.$ ape for some $\left.e \in E^{+}(S)\right\}$ is a normal full $k$-ideal of $S$. Moreover, the relation $\sigma$ on $S$ defined by

$a \sigma b$ if and only if $a+b^{*} \in H$ and $c a+(c b)^{*} \in H$ for all $c \in S$,

coincides with $\rho$. 
Theorem 3.19 ([1], corrected version) Let $(S,+,$.$) be an additively inverse semi-$ nearring. Let $\mathcal{I}_{\mathcal{R}}(S)$ be the set of all right normal congruences on $S$ and $\mathcal{J}_{\mathcal{R}}(S)$ be the set of all normal full right $k$-ideals of $S$. Then $\mathcal{I}_{\mathcal{R}}(S)$ and $\mathcal{J}_{\mathcal{R}}(S)$ are in an inclusion preserving bijective correspondence via the map $\phi: \rho \mapsto H_{\rho}$, where $H_{\rho}:=\left\{a \in S:\right.$ ape for some e $\left.\in E^{+}(S)\right\}$.

Theorem 3.20 ([1], corrected version) Let $(S,+,$.$) be a distributively generated$ additively inverse seminearring with property $D$. Let $\mathcal{I}_{\mathcal{C}}(S)$ be the set of all normal congruences on $S$ and $\mathcal{J}_{\mathcal{C}}(S)$ be the set of all normal full k-ideals of $S$. Then there exists an inclusion preserving bijection between $\mathcal{I}_{\mathcal{C}}(S)$ and $\mathcal{J}_{\mathcal{C}}(S)$ via the map $\phi: \rho \mapsto H_{\rho}$, where $H_{\rho}:=\left\{a \in S:\right.$ a $:$ for some e $\left.\in E^{+}(S)\right\}$.

Remark 3.23 ([1], corrected version) Theorem 3.22 together with Theorem 3.20 implies that $H_{\sigma}$ is the least normal full $k$-ideal of $S$.

\section{Reference}

1. Sardar, S.K., Mukherjee, R.: On additively regular seminearrings. Semigroup Forum 88(3), 541-554 (2014) 